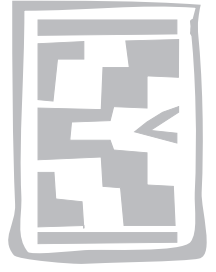

\title{
The effect of pyridoxal-5-phosphate on serum alanine aminotransferase activity in dogs suffering from canine babesiosis
}

\author{
E.C. MYBURGH and A. GODDARD* \\ Department of Companion Animal Clinical Studies, Section of Clinical Pathology, Faculty of Veterinary \\ Science, University of Pretoria, Private Bag X04, Onderstepoort, 0110 South Africa
}

\begin{abstract}
MYBURGH, E.C. \& GODDARD, A. 2009. The effect of pyridoxal-5-phosphate on serum alanine aminotransferase activity in dogs suffering from canine babesiosis. Onderstepoort Journal of Veterinary Research, 76:327-333

Accurate measurements of serum aminotransferase (ALT) activity in dogs relies on the endogenous pro-enzyme pyridoxal 5-phosphate (P5P). The purpose of this study was to determine whether the exclusion of P5P from the analytical method causes an underestimation of serum ALT activity in dogs suffering from babesiosis and in those manifesting evidence of hepatocellular damage, and to determine if anorexia causes sufficient P5P depletion to affect in vitro serum ALT activity. One-hundredand-twenty healthy control dogs and 105 Babesia-infected dogs were included in the study. Two methods for ALT measurement were used: Method 1 included P5P, and Method 2 excluded P5P from the reaction mixture. Higher serum ALT activity was measured with Method 1 in the Babesia-infected dogs $(P<0.001)$, as well as in 14 dogs with suspected hepatocellular damage $(P=0.03)$. Duration of anorexia had no effect, irrespective of the method used. Although inclusion of P5P to the reaction mixture consistently resulted in higher measured serum ALT activity, the differences were too small to have led to incorrect diagnoses in the Babesia-infected dogs suspected of liver disease.
\end{abstract}

Keywords: Alanine aminotransferase, anorexia, babesiosis, liver disease, pyridoxal 5'-phosphate

\section{INTRODUCTION}

Serum aminotransferases are enzymes that are often used to assist in the diagnosis of liver disease in domestic animals. Variable amounts of both aspartate aminotransferase (AST) and alanine aminotransferase (ALT) occur in the apo-enzyme form (inactive protein portion), which has no catalytic activity, and needs to be converted to an active holoenzyme form by the addition of pyridoxal 5-phosphate (P5P), the bio-active metabolite of dietary vitamin $B_{6}$ (Mesher, Rej \& Stokol 1998; Stokol \& Erb 1998). P5P is water-soluble and is excreted in the

* Author to whom correspondence is to be directed. E-mail: amelia.goddard@up.ac.za

Accepted for publication 16 March 2009-Editor urine; consequently, normal serum levels and cellular levels need to be maintained by daily dietary intake, and therefore may be affected by anorexia in patients. Measurements of the serum activity of these aminotransferases in mammalian patients with liver disease rely on the endogenous P5P level. In most patients, however, serum P5P is sufficient to allow the in vitro reaction to proceed optimally (Vinyard, Joven, Swendseid \& Drenick 1967).

Canine babesiosis, a tick-borne disease caused by the intra-erythrocytic protozoan parasites, Babesia rossi and Babesia vogeli, is a significant cause of morbidity in dogs in South Africa (Matjila, Leisewitz, Jongejan \& Penzhorn 2008). In a retrospective study by Reyers, Leisewitz, Lobetti, Milner \& Jacobson (1998), the serum ALT activity was measured in 662 
Pyridoxal-5-phosphate on serum alanine aminotransferase activity in dogs suffering from canine babesiosis

dogs that were diagnosed with canine babesiosis. For this study the haematocrit $(\mathrm{Ht})$ was used to classify the cases into three groups: severely anaemic $(\mathrm{Ht}<0.15 \mathrm{ll})$, moderately anaemic ( $\mathrm{Ht} 0.15-0.29 \mathrm{ll})$ and non-anaemic $(\mathrm{Ht}>0.30 \mathrm{ll})$ groups. The mean serum ALT activity was relatively high in the anaemic groups, but still within the normal reference limits. Only $41 \%$ of the dogs in the severely anaemic group and $25 \%$ of the dogs in the non-anaemic group had elevated serum ALT activity above the upper reference limit. The data from this study suggested that there may be an underestimation of serum ALT activity in the recorded babesiosis cases. It has been confirmed that human serum ALT and AST determination requires P5P for full activation (Horder \& Bowers 1977; Bergmeyer, Scheibe \& Wahlefeld 1978; Nanji 1983; Vanderlinde 1986; Rej, Rudolfsky, Magro \& Prendergast 1990).

Most routinely used kits do not include the addition of exogenous P5P to the reagent mixture. There are, however, analytical reagent kits available that do use added P5P in order to overcome the potential problem of P5P depletion in the patient. These methods require a two-step process and many automated analysers do not easily accommodate a two-step process. The incubation time for the two-step process is also longer and the ideal incubation time exceeds the programmable time limit of some instruments.

The objective of this study was to determine whether the use of the method, without addition of P5P, could lead to underestimation of ALT, leading to underdiagnosis of liver disease in dogs suffering from canine babesiosis, a disease that is often associated with inappetance and anorexia (Jacobson \& Clark 1994; Lobetti 1998; Jacobson 2006).

\section{MATERIALS AND METHODS}

\section{Study design}

Serum ALT activity of healthy control dogs and Babesia-infected dogs was assessed using two methods that differed with regards to the inclusion or exclusion of P5P in the reaction mixture: Method 1 with added P5P and Method 2 without P5P. The latter method is the most commonly used method in laboratories according to the Bio-rad Laboratories External Quality Assurance Services 2007. This study was done, primarily, to determine the effect of added exogenous P5P on the activation of serum ALT activity measurements and secondly, to determine the effect of anorexia (including its duration) on the underestimation of ALT activity levels of Babesiainfected patients using a non-P5P activated method.

\section{Experimental animals}

\section{Controls}

One-hundred-and-twenty dogs were used as controls. These were deemed clinically healthy (based on clinical and blood smear evaluation) and had no record or history of anorexia in the week prior to sample collection.

\section{Patients}

One-hundred-and-five dogs that had been diagnosed with babesiosis based on the demonstration of Babesia parasites (trophozoites) in erythrocytes on a thin blood smear, stained with a Diff Quick solution (Kyro-Quick stain, Kyron Laboratories (Pty) Ltd., Benrose, Johannesburg) were included. The babesiosis group was divided into five subgroups according to the number of days of anorexia:

Group 11 day of anorexia $(n=31)$

Group 22 days of anorexia $(n=30)$

Group 3 between 1-3 days of anorexia $(n=10$; excludes the first two subgroups)

Group 43 days of anorexia $(n=21)$

Group $5 \geq 4$ days of anorexia $(n=13)$.

The babesiosis group was also classified on the basis of the presence/absence of hepatocellular damage, due to decreased perfusion as seen in anaemia, based on their serum ALT activity: A serum ALT activity of $\geq 1.7 \times$ top normal (i.e. ALT $\geq 80 \mathrm{U} / \ell$, normal $17-47 \mathrm{U} / \mathrm{l}$ ) at admission, as measured by Method 2, was considered a reasonable indicator of the presence of hepatocellular damage $(n=14)$ (Jacobson 2006); and dogs without possible hepatocelluar damage $(n=91)$.

The reference limits used for serum ALT activity, as measured by the method without P5P (Clinical Pathology Laboratory, Faculty of Veterinary Science, University of Pretoria, Onderstepoort), were $17.0 \mathrm{U} / \ell$ to $47.0 \mathrm{U} / \ell$. This was taken from the Boehringer Mannheim range for dogs published for the DGKC-optimised reagent used in that laboratory between 1975 and 1990 (Schmid \& Von Forstner 1986).

\section{Blood analysis}

Serum ALT activity was measured using the standard methodological principle, namely the reduction of the oxo acid product with a specific dehydrogenase, in this case lactate dehydrogenase (LDH). 
The oxidation of $\mathrm{NADH}$ was then assessed spectrophotometrically at $340 \mathrm{~nm}$. The analyser, the Technicon RAXT (Bayer, South Africa), was maintained and calibrated according to the specifications of the manufacturer. Internal and external quality control samples were analysed in each analytical run. Method 1 involved the measurement of ALT with exogenous P5P added to the reagent solution. One tablet of pyridoxal 5-phosphate (0.3 mg P5P; Bayer catalogue number T01-1760-AO) was added to $10 \mathrm{ml}$ of ALT reagent prior to the analysis and allowed to dissolve. The sample volume used was $18 \mu \ell$ and the reagent volume $330 \mu \ell$, with a pre-incubation time of $5 \mathrm{~min}$ on the analyser at $37^{\circ} \mathrm{C}$, to allow for the activation of ALT by the exogenous P5P. A further incubation time of $1 \mathrm{~min}$ and $30 \mathrm{~s}$ was allowed for the standard reaction to take place. Method 2 involved the measurement of ALT without exogenously added P5P. All other test conditions (besides the $5 \mathrm{~min}$ pre-incubation period) remained the same as for Method 1. The shorter incubation time is regarded as one of the advantages of this method. All the test samples were analysed in the same analytical run to minimize analytical variation. Samples from the control dogs were also analysed in one analytical run but at a later stage.

\section{Statistical analysis}

The paired t-test was used to calculate the mean difference between all the observed activity readings for each case (controls and Babesia-infected dogs) for Method 1 versus Method 2. The coefficient of variation (CV) was calculated for Method 1 and Method 2 for dogs in the control group, the Babesia-infected group, and the group suspected of hepatocellular damage in the Babesia-infected group. In the Babesia-infected dogs, using the Bland-Altman analysis, the differences between the two methods (Method 1 minus Method 2) were plotted against the mean for each ALT reading from both methods [(Method 1 plus Method 2) divided by 2] as recommended by Altman \& Bland (1983) and Hopkins (2004) for the comparison of one method with another. The data were expressed in absolute units. The effect of the duration of anorexia in the Babesia-infected dogs was evaluated by linear regression analysis of the duration of anorexia on the difference in ALT activity between Method 1 and Method 2. For all comparisons, differences were considered significant when $P<0.05$.

\section{RESULTS}

\section{Serum ALT activity in control samples}

The serum ALT activity of all the control samples analysed by Method 1 was equal to or greater than that obtained by Method 2. The $95 \%$ confidence interval for Method 1 was 8.25-49.53 U/l $\left(\mathrm{x}_{1} \pm 2 \mathrm{SD}_{1}\right.$, $\left.\mathrm{SD}_{1}=10.32\right)$ with a mean $\left(\mathrm{x}_{1}\right)$ of $28.9 \mathrm{U} / \mathrm{l}$. For Method 2 the $95 \%$ confidence interval was 8.25-41.73 U/l $\left(\mathrm{x}_{2} \pm 2 \mathrm{SD}_{2}, \mathrm{SD}_{2}=8.36\right)$ with a mean $\left(\mathrm{x}_{2}\right)$ of $25.0 \mathrm{U} / \mathrm{l}$. $A$ difference of $3.9 \mathrm{U} / \ell$ between the mean values for the two methods was observed. Data were normally distributed. The CV for Method 1 was 0.36 and for Method 2 it was 0.33 . To the authors' knowledge, no reference ranges for serum ALT activity in dogs, with added P5P (Method 1), have been published to date. Although the difference in the mean serum ALT activity between the two methods was relatively small, it was, however, statistically significant $(P<$ 0.0001; paired t-test). The two methods did show a positive correlation $(r=0.981)$ (Fig. 1).

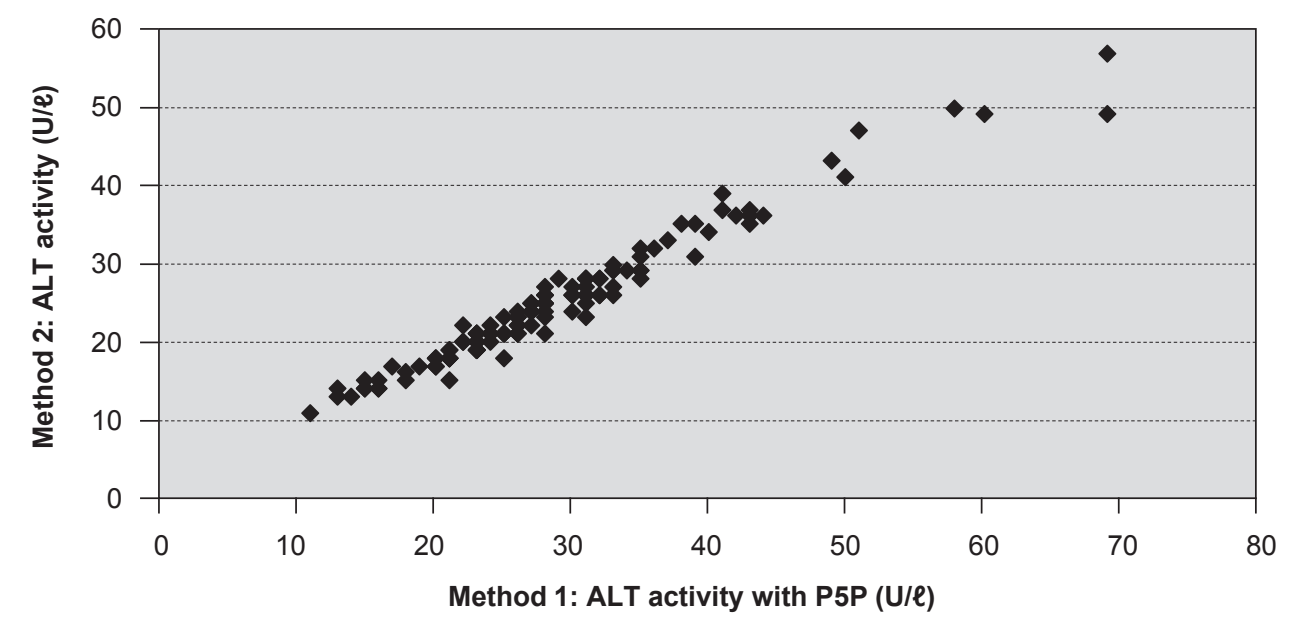

FIG. 1 Correlation of ALT measurements with P5P (Method 1) and ALT measurements without P5P (Method 2) in the control dogs 
Pyridoxal-5-phosphate on serum alanine aminotransferase activity in dogs suffering from canine babesiosis

The statistically significant increase in serum ALT activity in the control samples using Method 1, compared to those in Method 2, clearly indicates a beneficial effect of exogenous P5P on the accurate measurement of serum ALT activity. This increase is assumed to be because of the full saturation of the ALT apo-enzyme by the addition of exogenous P5P (Nanji 1983; Westerhuis \& Hafkenscheid 1983; Vanderlinde 1986; Rej et al. 1990)

\section{Serum ALT activity in the Babesia-infected dogs}

Most of the samples (86\%; 90/105) had higher serum ALT activity levels when analysed by Method 1 compared to Method 2. The mean difference between the two methods was relatively small $(4.2 \mathrm{U} / \ell)$, although statistically significant $(P<0.0001$; paired t-test). The CV for Method 1 was 2.79 and 2.83 for Method 2. The two methods showed a strong positive correlation, as was observed in the control dog samples (Fig. 2). Fifty-three percent (53\%; 56/105) of the infected dogs had serum ALT activity differences of $2 \mathrm{U} / \mathrm{l}$ or less, $28 \%(29 / 105)$ differed by between 3 and $5 \mathrm{U} / \ell$, and $17 \%(18 / 105)$ dogs had serum differences greater than $5 \mathrm{U} / \ell$. Only one dog had a difference of $15 \mathrm{U} / \ell$ while another dog differed by $96 \mathrm{U} / \ell$. This last dog had a serum ALT activity in excess of $1500 \mathrm{U} / \ell(1576 \mathrm{U} / \ell$ and $1480 \mathrm{U} / \ell$ with Methods 1 and 2, respectively) which is not shown in Fig. 2.

\section{Serum ALT activity in patients with suspected hepatocellular damage}

The mean difference between the two methods for the subset of Babesia-infected dogs with suspected hepatocellular damage $(n=14)$ was $13.8 \mathrm{U} / \ell$ (Table 1). This difference was statistically significant $(P=$ 0.0306 , paired t-test). The CV was 1.49 for both Method 1 and Method 2. The classification of the infected dogs into those suspected to be suffering from hepatocellular damage versus those which did not, using serum ALT activity, was unchanged by the use of either Method 1 or Method 2. The average difference between the two methods for this group of dogs was $15.2 \mathrm{U} / \ell(P=0.0306)$, but only four dogs $(28.6 \%)$ had differences greater than $10 \mathrm{U} / \ell$. In general, the sera from the infected dogs with suspected hepatocellular damage (i.e. ALT activity $\geq 80 \mathrm{U} / \ell$ with Method 2) showed consistently larger differences between the mean of the two methods $(13.8 \mathrm{U} / \ell)$ than in those assumed not to have hepatocellular damage (ALT activity $<80 \mathrm{U} / \ell$ with Method 2 ), which had a mean difference of $3.9 \mathrm{U} / \ell$.

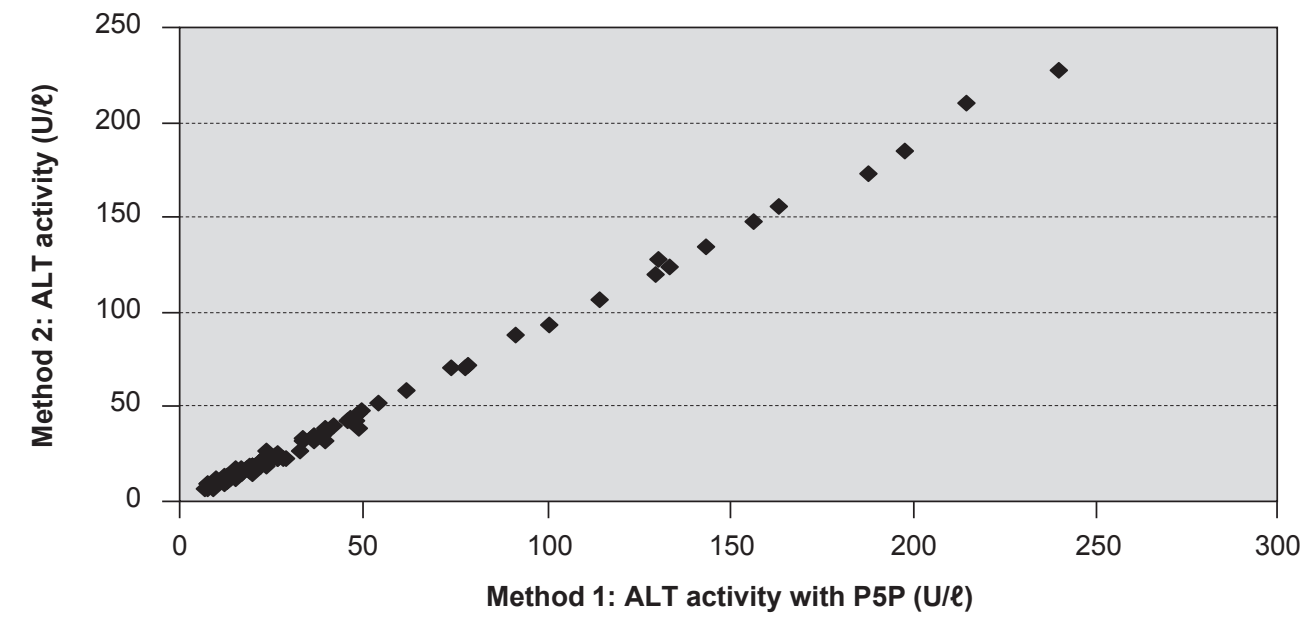

FIG. 2 Correlation of ALT measurements with P5P (Method 1) and ALT measurements without P5P (Method 2) in the dogs suffering from babesiosis

TABLE 1 Correlation between Method 1 and Method 2 in the Babesia-infected dogs with suspected hepatocellular damage $(n=14)$ where serum $A L T$ measured $\geq 1.7 x$ the upper limit of the normal value

\begin{tabular}{|l|l|l|l|l|}
\cline { 2 - 5 } \multicolumn{1}{l|}{} & $\begin{array}{l}\text { Method 1 } \\
\text { ALT with P5P }\end{array}$ & $\begin{array}{l}\text { Method 2 } \\
\text { ALT without P5P }\end{array}$ & t-value & $P$-value (paired t-test) \\
\hline Range & $92-1576 \mathrm{U} / \ell$ & $88-1480 \mathrm{U} / \ell$ & 2.43 & $P=0.0306$ \\
\hline Mean & $256.1 \mathrm{U} / \ell$ & $242.3 \mathrm{U} / \ell$ & & \\
\hline
\end{tabular}


TABLE 2 Correlation between Method 1 and Method 2 and the days of anorexia in the Babesia-infected dogs

\begin{tabular}{|l|l|l|l|}
\hline Anorexia & $\begin{array}{l}\text { Method 1 } \\
\text { ALT with P5P } \\
\text { (mean value) }\end{array}$ & $\begin{array}{l}\text { Method 2 } \\
\text { ALT without P5P } \\
\text { (mean value) }\end{array}$ & Paired t-test \\
\hline 1 day $(n=31)$ & $41.0 \mathrm{U} / \ell$ & $37.8 \mathrm{U} / \ell$ & $P<0.0001$ \\
\hline 2 days $(n=30)$ & $90.6 \mathrm{U} / \ell$ & $84.4 \mathrm{U} / \ell$ & $P=0.0235$ \\
\hline $1-3$ days $(n=10)$ & $37.1 \mathrm{U} / \ell$ & $33.8 \mathrm{U} / \ell$ & $P=0.0036$ \\
\hline 3 days $(n=21)$ & $46.4 \mathrm{U} / \ell$ & $42.9 \mathrm{U} / \ell$ & $P=0.0001$ \\
\hline $4+$ days $(n=13)$ & $44.1 \mathrm{U} / \ell$ & $40.5 \mathrm{U} / \ell$ & $P=0.0106$ \\
\hline
\end{tabular}

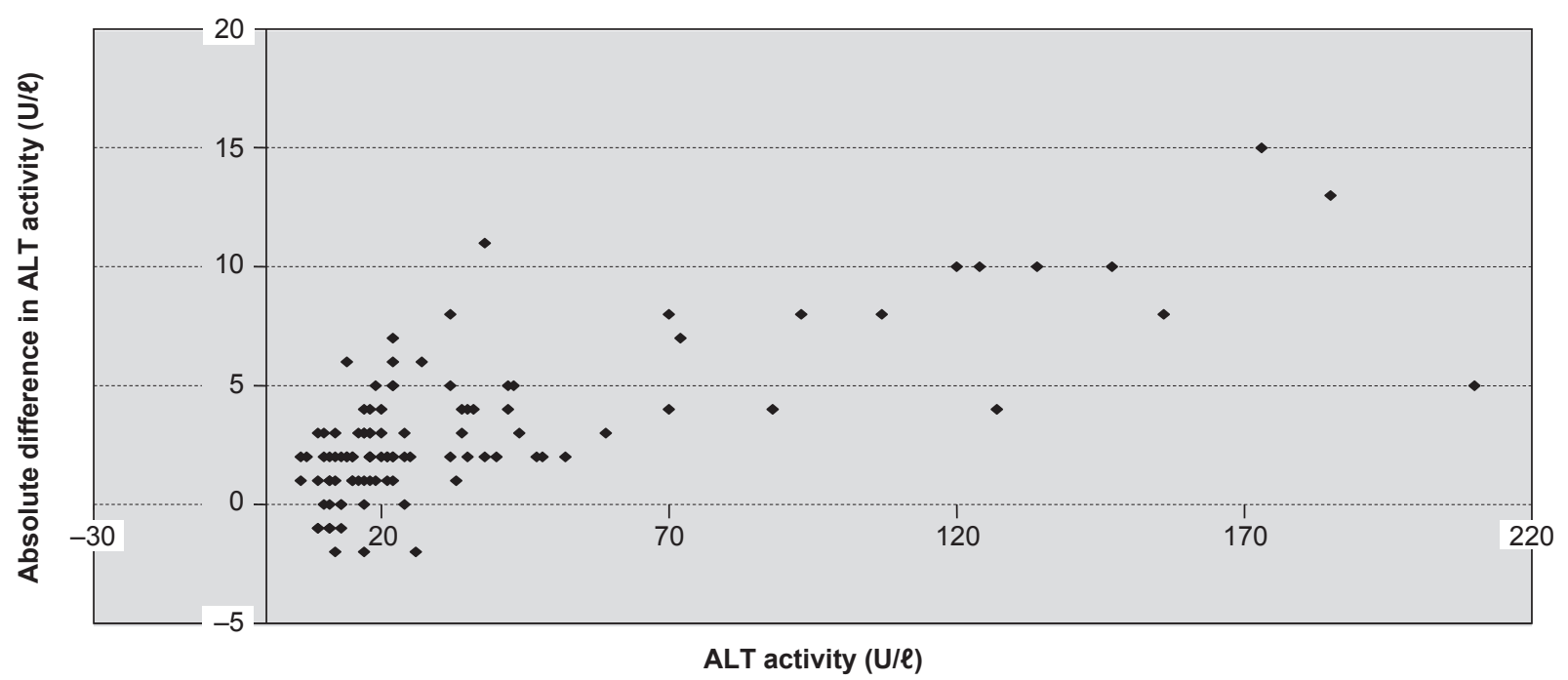

FIG. 3 The Bland-Altman absolute difference plot related to ALT activity in the Babesia-infected dogs

\section{Serum ALT activity in dogs with anorexia}

The serum ALT activity for dogs reported with periods of anorexia using Methods 1 and 2 are shown in Table 2. The increase in serum ALT activity, using Method 1, was statistically significant in all the groups of anorexic dogs. The extent of the difference between the methods did, however, not increase with the increase in the duration of anorexia. It was of interest to determine if this difference was progressively greater with each additional day of anorexia. The correlation was thus tested between the difference between Methods 1 and 2, and the number of days of anorexia (Table 2). There was no significant correlation or linear association between the days of anorexia and the depletion of P5P in canine babesiosis for up to 4 days $(r=0.0103)$.

\section{Bland-Altman Analysis}

The difference between Method 1 and Method 2, as determined by the Bland-Altman analysis, was al- most always positive in the Babesia-infected dogs (Fig. 3). The few negative differences were restricted to serum ALT activity of less than $25 \mathrm{U} / \ell$. For values above a serum ALT activity of $\pm 40 \mathrm{U} / \ell$, the increase in serum ALT activity, attributable to the effect of P5P, was constant as a proportion (or percentage) of the mean ALT activity, running at 2-9\% of the mean ALT activity (Fig. 3). This shows that P5P did activate the apo-enzyme, that the apo-enzyme was a constant proportion of the total serum $\mathrm{ALT}$, and that P5P concentration was not rate limiting.

\section{DISCUSSION}

The results of this study showed on average a $16 \%$ increase in serum ALT activity after the addition of P5P to the reagent mixture which, when compared with that in the published literature, is relatively small. 
Pyridoxal-5-phosphate on serum alanine aminotransferase activity in dogs suffering from canine babesiosis

Previous studies reported an increase in serum ALT activity of $17-49 \%$ in humans and $23-150 \%$ in animals (Marsh, Greenberg \& Rinehart 1955; Horder \& Bowers 1977; Bergmeyer et al. 1978; Vanderlinde 1986; Mesher et al.1998). In this study it is important to note that the addition of P5P to the serum of the dogs suffering from babesiosis did not have a clinically significant effect on the ALT activity to the degree that any of the animals would have been misclassified for the presence or absence of hepatocellular damage. The mean difference in ALT activity in the Babesia-infected dogs was similar to that obtained in the control dogs $(4.2 \mathrm{U} / \ell$ and $3.9 \mathrm{U} / \ell$, respectively). The difference was therefore not considered as clinically significant.

In this study the 14 dogs that had ALT activity higher than $80 \mathrm{U} / \ell$ measured consistently higher with Method 1 than with Method 2. An interpretation, in light of this study, is that the hepatocellular damage had an effect on the availability of P5P in the sera from these patients.

It appears that the presence of hepatocellular damage reduces the availability of $\mathrm{P} 5 \mathrm{P}$ and that the addition of P5P to the reaction mixture, therefore, enhances the serum ALT activity in such cases more than it does in patients without hepatocellular damage. However, one could speculate that there may be other explanations such as the presence of inhibitors released by damaged hepatocytes or produced during the inflammatory reaction. Neither of these potential explanations could be confirmed on the basis of the data available from this study or from the hospital case records. The data did confirm that the activation of canine serum ALT by P5P is significant in patients in which hepatocellular damage is presumed to be present, based on elevated serum ALT activity. The difference in activity between the two methods is, however, unlikely to lead to an incorrect clinical conclusion regarding the presence of liver disease.

The length of anorexia, as reported by the owners, had no significant effect on the measured ALT activity as the difference between the two methods did not increase with an increase in the duration of anorexia. In one dog, with a serum ALT of $1576 \mathrm{U} / \ell$ (Method 1) and a serum ALT of $1480 \mathrm{U} / \ell$ (Method 2 ), one may assume that the severe depletion of serum P5P was the cause for the difference in serum ALT activity. As this specific case was only reported to be anorexic for 2 days, the P5P depletion could have been due to another cause such as a concurrent or pre-existing disease. Unfortunately, this could not be confirmed since no further testing was done on this case. It is, however, possible that the length of anorexia, exhibited by these patients (up to 5 days) was not long enough to deplete the endogenous body reserves of P5P. This suggests that the sera from the greatest majority of dogs with babesiosis in this study were not severely depleted of P5P, and implies that a large reserve capacity may exist for P5P or, possibly, that mild or moderate depletion did not represent a rate-limiting factor in the reaction as suggested by Conzolazio, Johnson, Krzywicki, Daws \& Barnart (1971) in humans. Further studies are needed to investigate the serum ALT activity with and without P5P in illnesses with longer periods of anorexia.

The Bland-Altman technique of method comparisons (Method 1 versus Method 2) revealed that there was a true enhancement of the activity of serum ALT by adding P5P and that this effect was progressively greater, the higher the serum activity of ALT. Furthermore, the analysis provided evidence that it was, in fact, the P5P that enhanced the activity. The percentage difference between the methods was relatively high between serum activities of $0 \mathrm{U} / \ell$ and $40 \mathrm{U} / \ell$, but then decreased to a fairly constant percentage value. The reasonable conclusion to be drawn from this is that the proportion of canine serum ALT apo-enzyme that can be activated is roughly constant, as a proportion of the total serum ALT enzyme. The relatively high percentage difference at lower activities (below $25 \mathrm{U} / \ell$ ) allows one to conclude that, at this level of serum ALT activity, the methodological background random error is larger (on average) than the effect of the P5P activation.

The effect of added P5P on serum ALT activity in dogs suffering from babesiosis was examined in this study. It was found that, although there was a statistically significant increase in ALT activity with added exogenous P5P, it was unlikely to lead to incorrect clinical diagnoses of hepatocellular damage in patients, suffering from liver disease.

\section{ACKNOWLEDGEMENTS}

This study was carried out in partial fulfillment of the requirements for the Magister Technologiae: Biomedical Technology, Tswhane University of Technology, South Africa. Funding was received from the Faculty of Veterinary Science, University of Pretoria, Onderstepoort and Bayer Health Care, South Africa. The work was done at the Onderstepoort Veterinary Academic Hospital, Faculty of Veterinary Science, University of Pretoria, South Africa. 


\section{REFERENCES}

ALTMAN, D.G. \& BLAND, J.M. 1983. Measurement in medicine: the analysis of method comparison studies. The Statistician, 32:307-317.

BERGMEYER, H.U., SCHEIBE, P. \& WAHLEFELD, A.W. 1978. Optimization of methods for aspartate aminotransferase and alanine aminotransferase. Clinical Chemistry, 24:58-73.

CONSOLAZIO, C.F., JOHNSON, H.L., KRZYWICKI, H.J., DAWS, T.A. \& BARNHART, R.A. 1971. Thiamin, riboflavin, and pyridoxine excretion during acute starvation and calorie restriction. American Journal of Clinical Nutrition, 24:1060-1067.

HOPKINS, W.G. 2004. Bias in Bland-Altman but not regression validity analyses. Sportscience, 8:42-46.

HORDER, M. \& BOWERS, G.N. 1977. Biological variability in aspartate aminotransferase activity in serum of healthy persons, and effect of in vitro supplementation with pyridoxal 5-phosphate. Clinical Chemistry, 23:551-554.

JACOBSON, L.S. \& CLARK, I.A. 1994. The pathophysiology of canine babesiosis: New approaches to an old puzzle. Journal of the South African Veterinary Association, 65:134-145.

JACOBSON, L.S. 2006. The South African form of severe and complicated canine babesiosis: Clinical advances 1994-2004. Veterinary Parasitology, 138:126-139.

LOBETTI, R.G. 1998. Canine babesiosis. Compendium of Continuing Education, 20:418-430.

MARSH, M.E., GREENBERG, C.D. \& RINEHART, J.F. 1955. The relationship between pyridoxine ingestion and transaminase activity. Journal of Nutrition, 56:115.

MATJILA, P.T., LEISEWITZ, A.L., JONGEJAN, F. \& PENZHORN, B.L. 2008. Molecular detection of tick-borne protozoal and ehrlichial infections in domestic dogs in South Africa. Veterinary Parasitology, 155:152-157.
MESHER, C.I., REJ, R. \& STOKOL, T. 1998. Alanine aminotransferase apoenzyme in dogs. Veterinary Clinical Pathology, 27:26-30.

NANJI, A.A. 1983. Decreased activity of commonly measured serum enzymes: Causes and clinical significance. American Journal of Medical Technology, 49:241-245.

REJ, R., RUDOFSKY, U., MAGRO, A. \& PRENDERGAST, J. 1990. Effects of exercise on serum amino-transferase activity and pyridoxal phosphate saturation in Thoroughbred racehorses. Equine Veterinary Journal, 22:205-208.

REYERS, F., LEISEWITZ, A.L., LOBETTI, R.G., MILNER, R.J. \& JACOBSON, L.S. 1998. Canine babesiosis in South Africa: more than one disease. Does this serve as a model for falciparum malaria? Annals of Tropical Medicine \& Parasitology, 92:503-511.

STOKOL, T. \& ERB, H. 1998. The apo-enzyme content of aminotransferases in healthy and diseased domestic animals. Veterinary Clinical Pathology, 27:71-78.

SCHMID, M. \& VON FORSTNER, L. 1986. Laboratory testing in veterinary medicine diagnosis and clinical monitoring, $3^{\text {rd }}$ ed. Mannheim: Boehringer Mannheim GmbH.

VANDERLINDE, R.E. 1986. Review of pyridoxal phosphate and the transaminases in liver disease. Annals of Clinical and Laboratory Science, 16:79-93.

VINYARD, E., JOVEN, C.B., SWENDSEID, M.E. \& DRENICK, E.J. 1967. Vitamin B6 nutriture studied in obese subjects during 8 weeks of starvation. The American Journal of Clinical Nutrition, 20:317-323.

WESTERHUIS, L.W.J.J.M. \& HAFKENSCHEID, J.C.M. 1983. Apoenzyme content of serum aminotransferases in relation to plasma pyridoxal-5-phosphate concentration. Clinical Chemistry, 29:789-792. 